\title{
EFFECTS OF GRAIN SIZE ON CYCLIC STRAIN LOCALIZATION IN POLYCRYSTALLINE NICKEL
}

\author{
D.J. Morrisont, V. Choprat, and J.W. Jones+ \\ tDepartment of Mechanical and Aeronautical Engineering, Clarkson University, \\ Potsdam, NY 13699, +Department of Materials Science and Engineering, \\ The University of Michigan, Ann Arbor, Ml 48109
}

(Received December 11,1990 )

(Revised March 19, 1991)

\section{Introduction}

Substantial research has been conducted on cyclic plasticity and the accumulation of fatigue damage in copper and nickel single crystals. As a result, a rather comprehensive description of fundamental cyclic deformation processes in monocrystalline fcc materials has been developed (1-4). Far less fundamental work has been accomplished on polycrystalline materials, and more significantly, very few studies on the effects of grain size on cyclic plasticity and fatigue damage accumulation have been conducted.

The effect of grain size on monotonic stress-strain behavior can be described through models such as the Hall-Petch relationship. However, the effect of grain size on cyclic stress-strain behavior has not been as clearly defined. Lukás and Kunz (5) and Liang and Laird (6) found that at low strain amplitudes, the saturation stress of copper increased as grain size increased. However, in the Lukás and Kunz study, at higher strains the fine grain copper exhibited a slightly higher saturation stress. Mughrabi and Wang (7) studied the cyclic stress-strain response of polycrystalline copper with grain sizes ranging from $25 \mu \mathrm{m}$ to $400 \mu \mathrm{m}$ and found that grain size had very little effect on cyclic stress-strain response. They found a slightly higher saturation stress for coarse grain copper, but attributed the increase to a "hard" $\langle 111\rangle-\langle 100\rangle$ fiber texture that was present in the coarse grain material. Several other studies on materials other than nickel or copper have been reported. Horibe et al. (8) found that coarse grain Al-4\%Cu specimens exhibited a higher saturation stress than fine grain material. Boutin et al.' (9) presented evidence that in $\alpha$ brass, fine grain specimens exhibited a higher saturation stress than coarse grain specimens; and Lucas and Gerberich (10) found a similar grain size effect in microalloyed steels. It is clear from the preceding discussion that the questions concerning the role of grain size on cyclic plasticity remain to be answered.

Previous studies have shown that the localization of plastic strain in persistent slip bands (psbs) causes pronounced changes in the shape of the hysteresis loop (11-17). Two parameters that are commonly used to quantify the shape of the hysteresis loop are the loop shape parameter, $V_{H}$, defined as the area enclosed by the loop divided by the area of the circumscribing parallelogram (17), and the hardening coefficient, $H$, defined as the slope of the hysteresis loop, $d \sigma / d \varepsilon$, at the tension tip (14). Strain localization in psbs leads to a more parallelogram-shaped hysteresis loop which causes an increase in $V_{H}$ and decrease in $H$. The objective of the present study was to investigate the effects of grain size on strain localization during cyclic deformation of nickel. This was accomplished by determining the cyclic hardening coefficients and loop shape parameters for grain sizes which differed by approximately an order of magnitude. 


\section{Experimental Methods}

Cylindrical fatigue specimens were machined from nickel-270 $(99.98 \% \mathrm{Ni})$ which was received in the form of $12.5 \mathrm{~mm}$ diameter hot-rolled rods and $9.5 \mathrm{~mm}$ diameter cold-drawn rods. Specimens machined trom the cold-drawn rods were annealed in a flowing argon-3\% hydrogen atmosphere at $500^{\circ} \mathrm{C}$ for three hours to produce a microstructure with an average lineal grain boundary intercept of $24 \mu \mathrm{m}$. These specimens will be referred to as the fine grain specimens. Specimens machined from the hot-rolled rods were annealed in the same atmosphere for four hours at $1000^{\circ} \mathrm{C}$, resulting in a grain structure with an average lineal intercept of $290 \mu \mathrm{m}$. These specimens will be referred to as the coarse grain specimens. Twin boundaries were counted along with grain boundaries in lineal intercept measurements. Following heat treatment, the gauge sections of the specimens were electropolished in a solution of ethanol - $20 \%$ perchloric acid at $45 \mathrm{~V}$ and $-50^{\circ} \mathrm{C}$. The gauge sections were $15 \mathrm{~mm}$ long and $5 \mathrm{~mm}$ in diameter.

Cyclic deformation experiments were conducted at room temperature under fully-reversed tension/compression at a constant plastic strain amplitude $\left(\varepsilon_{\mathrm{p}}\right)$ of $2.5 \times 10^{-4}$. Prior to saturation, the specimens were cycled using a triangular waveform at a constant total strain rate of $2.0 \times 10^{-3} / \mathrm{s}$. After saturation the total strain rate was increased to $4.0 \times 10^{-2} / \mathrm{s}$. Hysteresis loops were periodically digitally recorded at a total strain rate of $2.0 \times 10^{-4} / \mathrm{s}$. The different strain rates did not produce noticeable changes in the stress-strain response. Parameters describing the shapes of the hysteresis loops were computed from the digitally recorded data after the completion of each fatigue experiment. The area enclosed by each hysteresis loop was computed using a numerical integration method. The area of the circumscribing parallelogram was determined by multiplying the stress range $\left(\sigma_{\max }-\sigma_{\min }\right)$ by the plastic strain range $\left(\varepsilon_{p, \max }-\varepsilon_{p, \min }\right)$. Hardening coefficients were determined by performing linear regression on the portion of the hysteresis loop that extended from $75 \%$ of the peak tensile total strain value to the tension tip.

\section{Besults and Discussion}

Cyclic hardening and loop shape parameter curves for the coarse grain specimen are shown in Figure 1. The cyclic hardening curve indicates that the specimen hardened to a peak stress amplitude of $160 \mathrm{MPa}$ at a cumulative plastic strain of 7 and then softened to about $151 \mathrm{MPa}$. The specimen failed at a cumulative plastic strain of 45 (45,000 cycles) as shown by the sudden drop in the stress amplitude. The loop shape parameter curve shows an initially low value of $V_{H}$ of about 0.67 . At a cumulative plastic strain of $1.0, V_{H}$ increased abruptly and reached a maximum value of 0.76 at a cumulative plastic strain of 10 .

To provide a fundamental basis of comparison, cyclic hardening and loop shape parameter curves for monocrystalline nickel oriented for single slip are shown in Figure 2. The data for this figure are from the work of Blochwitz and Veit (13). It is evident that the coarse grain polycrystalline and monocrystalline nickel exhibit similar loop shape behavior. However, the magnitude of the increase in $V_{H}$ is greater in the monocrystal. The monocrystal saturated at about $52 \mathrm{MPa}$ which corresponds to the plateau shear stress of the nickel cyclic stress-strain curve (18). The minimum $V_{H}$ occurred just prior to saturation. The abrupt increase of $V_{H}$ in the coarse grain material occurred when the specimen cyclically hardened to a stress amplitude of approximately $100 \mathrm{MPa}$. This stress is approximately equal to the monocrystal plateau shear stress (52 MPa) divided by the maximum Schmid factor (0.5). Therefore, psbs first form in grains oriented with a Schmid factor of 0.5 and cause a corresponding increase in $V_{H}$. This result is in agreement with previous discussions on the onset of psb formation in polycrystalline copper (1). 
Cyclic hardening and loop shape parameter curves for the fine grain specimen are shown in Figure 3. The specimen cyclically hardened to a peak stress amplitude of $178 \mathrm{MPa}$ at a cumulative plastic strain of 10 and then softened to a stress amplitude of $170 \mathrm{MPa}$. The loop shape parameter curve for the fine grain specimen reached a minimum value of 0.67 at a cumulative plastic strain of 1.0 and then increased to a maximum value of 0.70 at a cumulative plastic strain of 20 . As was observed in the coarse grain nickel, the minimum in the fine grain loop shape parameter occurred when the specimen hardened to a stress amplitude of approximately $100 \mathrm{MPa}$.

The fine grain nickel cyclically saturated at a higher stress amplitude than the coarse grain material, and the abrupt increase in the loop shape parameter was much less pronounced in the fine grain material. It is reasonable to infer from these bulk property data that the localization of plastic strain in psbs is not as extensive in the fine grain nickel. Even though the magnitudes of grain boundary compatibility strains would be expected to be larger in the coarse grain material, it is possible that the volume fraction of material influenced by compatibility conditions is greater in a fine grain material as a result of the much larger specific grain boundary surface area. Multiple slip induced by the compatibility strains should inhibit the formation of psbs and induce a greater degree of cyclic hardening. As a result, a fine grain material would demonstrate a lower level of strain localization and a higher saturation stress. It should also be noted that other factors such as the ratio of surface to interior grains and texture differences between fine and coarse grain materials may influence strain localization and cyclic hardening behavior. Texture determinations of the materials used in this study are currently being performed.

Grain refinement had a considerable effect on fatigue life. The fine grain specimen failed after 220,000 cycles compared to 45,000 for the coarse grain specimen. Similar grain size effects on fatigue life have been reported in copper $(6,7)$. This effect has been attributed to the more damaging effect of psb impingement on grain boundaries in course grain materials $(7,19,20)$. In the present study, intergranular crack initiation was observed in the coarse grain nickel while transgranular cracks were more commonly seen in the fine grain material.

It is interesting to note that in a study of grain size effects on fatigue of polycrystalline copper, Liang and Laird (6) reported similar influences of grain size on fatigue lifetime and crack initiation mode. However, visual comparisons of slip band geometry and density for coarse and fine grain materials magnified to the same relative size indicated that strain localization in psbs was greater in the fine grain material. From this observation, they concluded that transgranular crack initiation in fine grain material is expected because of the greater degree of strain localization. Further studies are required to explain the differences between surface and bulk deformation behavior and the effects of surface and bulk strain localization phenomena on crack initiation mode.

Hardening coefficients for the fine and coarse grain specimens are shown in Figure 4. The hardening coefficients reflect the same phenomena which affect loop shape parameters. The onset of plastic strain localization and the formation of psbs causes a decrease in the hardening coefficient. The extent of the decrease of the hardening coefficient is more pronounced in the coarse grain nickel, indicating a higher degree of strain localization in the coarse grain material. One notable difference between the loop shape parameter and hardening coefficient curves is that the hardening coefficient reaches a maximum slightly later than the minimum in the loop shape parameter curve. In monocrystalline studies, the peak hardening coefficient coincided with the minimum of the loop shape parameter $(14,15)$. 


\section{Conclusions}

From the results of these fatigue experiments on polycrystalline nickel cycled at a plastic strain amplitude of $2.5 \times 10^{-4}$, it can be concluded that grain refinement reduces the degree of bulk cyclic strain localization in psbs and increases fatigue life. In addition, in this study fine grain nickel exhibited a higher cyclic saturation stress than coarse grain nickel. It is possible that the magnitude of the difference in saturation stress for fine and coarse grain material is influenced by crystallographic texture. This possibility is currently being investigated.

\section{Acknowledaments}

Portions of this work were supported by the National Science Foundation through grant DMR 8603174 and by the U.S. Air Force Office of Scientific Research through an Air Force Engineering Research Initiation grant administered by the Engineering Foundation in cooperation with the American Institute of Mining, Metallurgical, and Petroleum Engineers.

\section{Beferences}

1. C. Laird and L. Buchinger, Metall. Trans. A, 16A, 2201-2214 (1985).

2. C. Laird, P. Charsley and H. Mughrabi, Mater. Sci. Eng., $81,433-450$ (1986).

3. H. Mughrabi, Proceedings of the 7th International Conference on the Strength of Metals and Alloys, H. J. McQueen, J. P. Bailon, J. I. Dickson, J. J. Jonas, and M. G. Akben, Eds., Pergamon, 1985, pp.1917-1942.

4. D. Kuhimann-Wilsdorf, Mater. Sci. Eng., 86, 53-66 (1987).

5. P. Lukás and L. Kunz, Mater. Sci. Eng., 85, 67-75 (1987).

6. F.-L. Liang and C. Laird, Mater. Sci. Eng., A117, 95-102 (1989).

7. H. Mughrabi, and R. Wang, in Basic Mechanisms in Fatigue of Metals. Materials Science Monographs, Vol 46, P. Lukás and J. Polák Eds., Elsevier, 1988, pp. 1-14.

8. S. Horibe, J. K. Lee, and C. Laird, Fat. Eng. Mater. Struc., 7, 145-154 (1984).

9. J. Boutin, N. Marchand, J. P. Bailon, and J. I. Dickson, Mater. Sci. Eng., 67, L23-L27 (1984).

10. J. P. Lucas and W. W. Gerberich, Int. J. Fat., 7, 31-38 (1985).

11. H. Mughrabi, Mater. Sci. Eng., 33, 207-223 (1978).

12. A. Abel, Mater. Sci. Eng., 36, 117-124 (1978).

13. C. Blochwitz and U. Veit, Crystal Res. and Technol., 17, 529-551 (1982).

14. J. Polák, J. Helesic and K. Obrtlík, Mater. Sci. Eng. A, 101, 7-12 (1988).

15. J. Polák, J. Helesic and K. Obrtlik, Scripta Metall., 24, 415-420 (1990).

16. D.J. Morrison, J.W. Jones and G.S. Was, Scripta Metall., 24, 2309-2314, (1990).

17. P. Schützner, Doctoral Thesis, Stuttgart University, 1974.

18. H. Mughrabi, F. Ackermann, and K. Herz, in ASTM STP 675, J. T. Fong, Ed., American Society for Testing and Materials, 1979, pp. 69-105.

19. H. Mughrabi, in Proceedings of the Second International Symposium on Defects, Fracture, and Fatigue, G.C. Sih and J.W. Provan, Eds., Martinus Nijhoff, 1983, pp. 139-146.

20. H.-J. Christ, Mater. Sci. Eng., A117, L25-L29 (1989). 


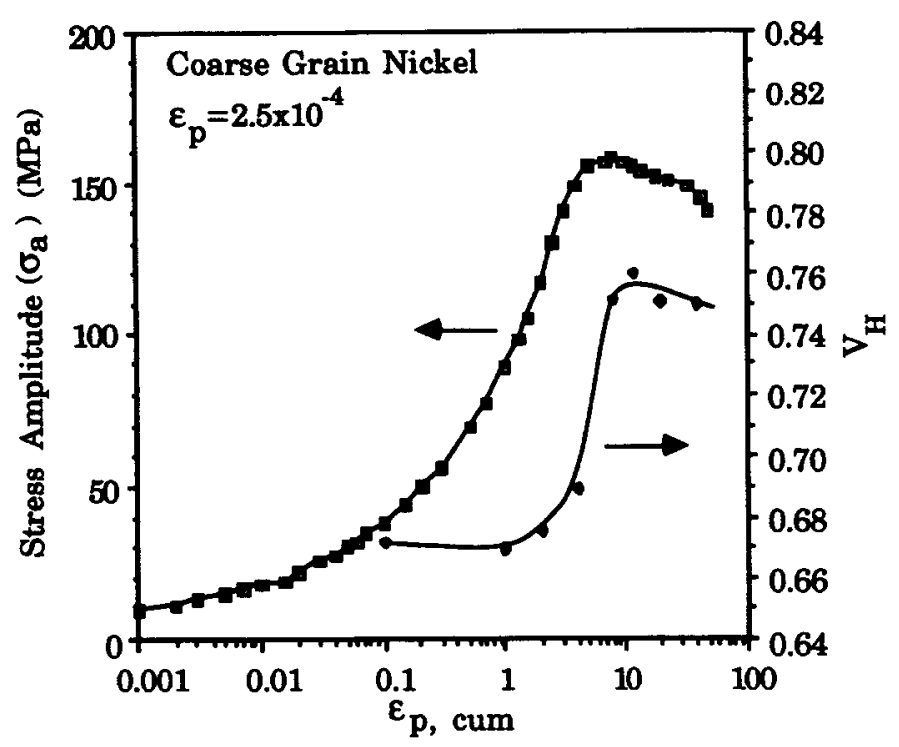

FIGURE 1. Cyclic hardening and loop shape parameter curves for coarse grain $(290 \mu \mathrm{m})$ nickel.

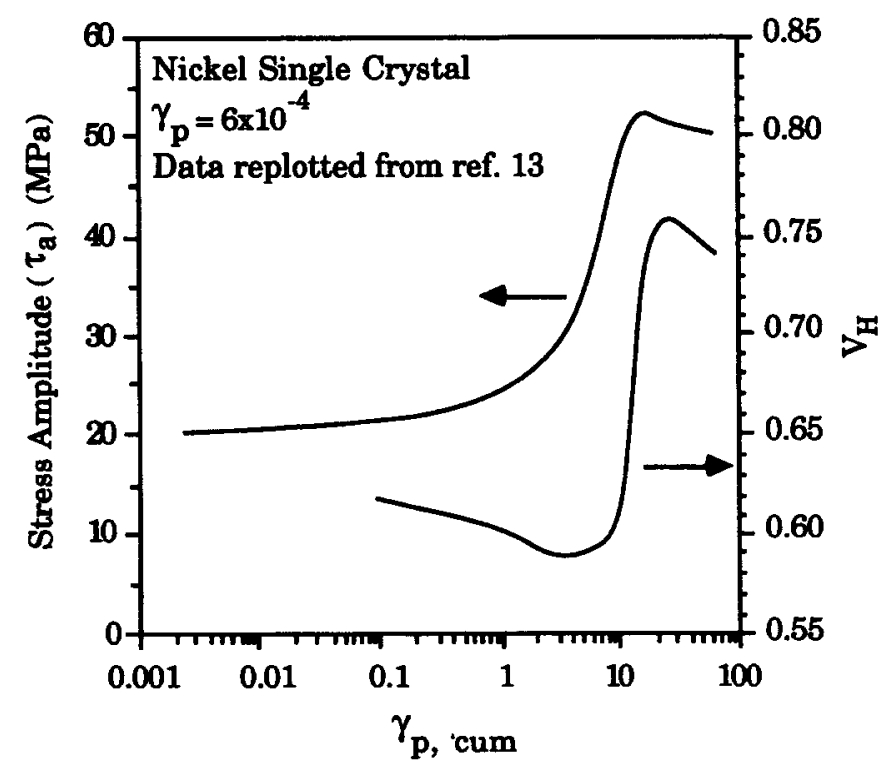

FIGURE 2. Cyclic hardening and loop shape parameter curves for monocrystalline nickel from the work of Blochwitz and Veit (13). 


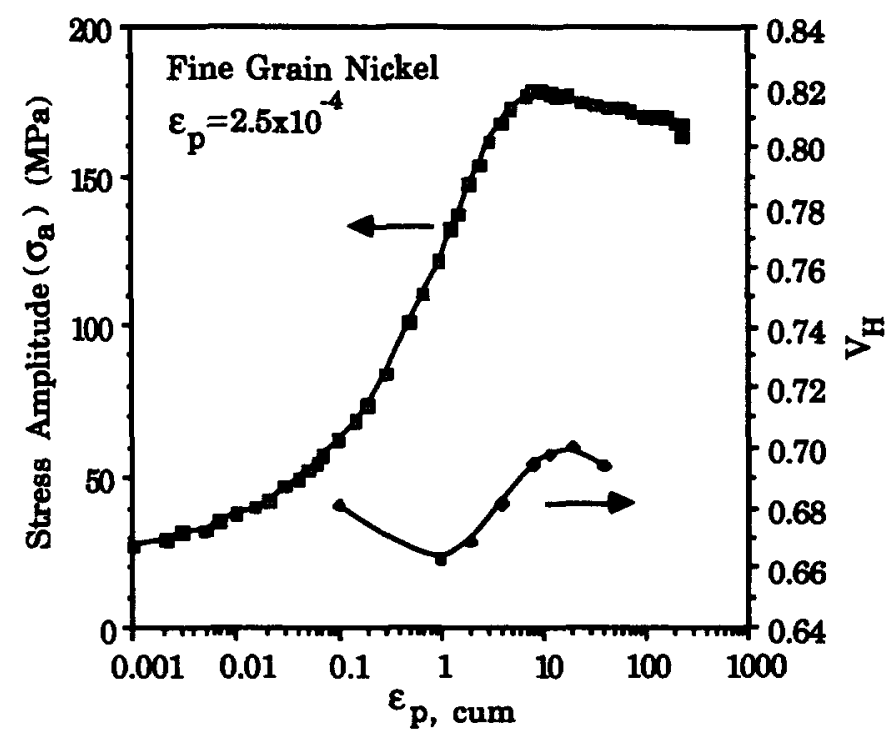

FIGURE 3. Cyclic hardening and loop shape parameter curves for fine grain (24 $\mu \mathrm{m})$ nickel.

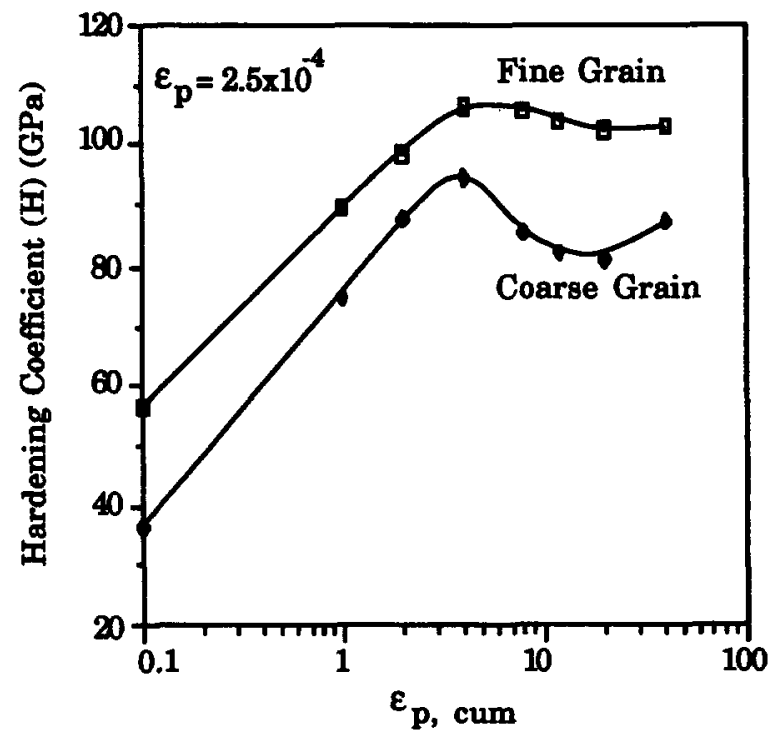

FIGURE 4. Hardening coefficient curves for coarse and fine grain nickel. 